\title{
Mutations in the cardiac transcription factor NKX2.5 affect diverse cardiac developmental pathways
}

\author{
D. Woodrow Benson, ${ }^{1}$ G. Michael Silberbach, ${ }^{2}$ Ann Kavanaugh-McHugh, ${ }^{3}$ \\ Carol Cottrill, ${ }^{4}$ Yizhong Zhang, ${ }^{1}$ Steve Riggs, ${ }^{1}$ Octavia Smalls, ${ }^{1}$ Mark C. Johnson, ${ }^{5}$ \\ Michael S. Watson, ${ }^{6}$ J.G. Seidman, ${ }^{7}$ Christine E. Seidman, ${ }^{7}$ John Plowden, ${ }^{8}$ \\ and John D. Kugler ${ }^{9}$
}

\begin{abstract}
${ }^{1}$ Division of Pediatric Cardiology, Medical University of South Carolina, Charleston, South Carolina 29425, USA
${ }^{2}$ Division of Pediatric Cardiology, UHN 60 - Oregon Health Sciences University, Portland, Oregon 97201, USA

${ }^{3}$ Division of Pediatric Cardiology, Vanderbilt University Medical Center, Nashville, Tennessee 37232, USA

${ }^{4}$ Division of Pediatric Cardiology, University of Kentucky, Lexington, Kentucky 40536, USA

${ }^{5}$ Division of Pediatric Cardiology, and

${ }^{6}$ Division of Medical Genetics, Department of Pediatrics, Washington University, St. Louis, Missouri 63110, USA

${ }^{7}$ Department of Genetics, Howard Hughes Medical Institute, Boston, Massachusetts 02115, USA

${ }^{8}$ Division of Pediatric Cardiology, University of New Mexico, Albuquerque, New Mexico 87131, USA

${ }^{9}$ Division of Pediatric Cardiology, University of Nebraska and Creighton University, Omaha, Nebraska 68198, USA

Address correspondence to: D. Woodrow Benson, Cardiovascular Genetics, Pediatric Cardiology, Medical University of South Carolina, 165 Ashley Avenue, PO Box 250915, Charleston, South Carolina 29425, USA.

Phone: (843) 792-8997; Fax: (843) 792-3284; E-mail: bensondw@musc.edu.
\end{abstract}

Received for publication August 13, 1999, and accepted in revised form October 12, 1999.

\begin{abstract}
Heterozygous mutations in $N K X 2.5$, a homeobox transcription factor, were reported to cause secundum atrial septal defects and result in atrioventricular (AV) conduction block during postnatal life. To further characterize the role of NKX2.5 in cardiac morphogenesis, we sought additional mutations in groups of probands with cardiac anomalies and first-degree AV block, idiopathic AV block, or tetralogy of Fallot. We identified 7 novel mutations by sequence analysis of the NKX2.5-coding region in 26 individuals. Associated phenotypes included AV block, which was the primary manifestation of cardiac disease in nearly a quarter of affected individuals, as well as atrial septal defect and ventricular septal defect. Ventricular septal defect was associated with tetralogy of Fallot or doubleoutlet right ventricle in 3 individuals. Ebstein's anomaly and other tricuspid valve abnormalities were also present. Mutations in human NKX2.5 cause a variety of cardiac anomalies and may account for a clinically significant portion of tetralogy of Fallot and idiopathic AV block. The coinheritance of NKX2.5 mutations with various congenital heart defects suggests that this transcription factor contributes to diverse cardiac developmental pathways.
\end{abstract}

J. Clin. Invest. 104:1567-1573 (1999).

\section{Introduction}

Transcription factors are increasingly recognized as having key roles in the complex biological processes governing cardiac development (1-3). The homeobox transcription factor, tinman, a Drosophila NK-gene, has been of particular interest because it is expressed in the dorsal vessel, an insect equivalent of the vertebrate heart. Targeted disruption of tinman results in the absence of the dorsal vessel (4).

A murine Nkx2.5 homeobox gene (5), also independently described as a cardiac-specific homeobox gene (CSX; see ref. 6), is a vertebrate homologue of tinman. $N k \times 2.5$ is expressed in early cardiac mesoderm and in heart muscle lineage throughout life (5-7); targeted disruption is lethal to embryos and arrests cardiac development at the linear heart tube stage $(8,9)$. Molecular dissection of $N k \times 2.5$ revealed the participation of highly conserved regions of Nkx2.5 in DNA binding, protein-protein interactions, nuclear translocation, and regulation of other transcription factors (9-18).
We recently described 3 heterozygote mutations in NKX2.5 that resulted in atrioventricular (AV) conduction block. Many genotype-positive individuals also had a secundum atrial septal defect (ASD) (19). In kindreds with NKX2.5 mutation, there was a history of other congenital heart malformations in some family members including ventricular septal defect and tetralogy of Fallot (TOF). To further characterize the role of NKX2.5 in human cardiac morphogenesis, we have identified additional mutations and defined the clinical phenotypes produced by these mutations.

In this report, 7 additional $N K X 2.5$ mutations are described. As reported previously, AV conduction abnormalities and ASD occurred commonly (19). However, we also found evidence of several different types of ventricular septal defects (VSD) that were sometimes associated with double-outlet right ventricle and tetralogy of Fallot. In addition, tricuspid valve abnormalities, including Ebstein's anomaly, were noted in some individuals. These observations illus- 
Table 1

NKX2.5 primers

$\begin{array}{lcc}\text { Primer sequence 5' to 3' } & \text { ID } & \text { Length } \\ \text { GTCCCGCCTCTCCTGCCCCTTGTG } & 1 \mathrm{~F} & 24 \\ \text { TCCTCCTCCTGGCCCTGAGTTCT } & 1 \mathrm{R} & 24 \\ \text { TGGGCGCTCCAGGCAGGACACAGT } & 2 \mathrm{~F} & 24 \\ \text { GCTTGCCATCGCGCACCAGCACTG } & 2 \mathrm{R} & 24 \\ \text { GTCCAGAACCGGCGCTACAAGTG } & 3 \mathrm{~F} & 24 \\ \text { GCGCGTGGGACAGAAAAAGTTCCT } & 3 \mathrm{R} & 24 \\ \text { GCGTGCCCGAGCTCAGTCCCAGTT } & 4 \mathrm{R} & 24 \\ \text { CGCAGGCGCAGGTCTAT } & \text { BEA-WT } & 17 \\ \text { CGCAGGCGTAGGTCTAT } & \text { BEA-MUT } & 17 \\ \text { CCGGCGCTACAAGTGCA } & \text { BEP-WT } & 17 \\ \text { CCGGCGCTGCAAGTGCA } & \text { BEP-MUT } & 17\end{array}$

trate an essential role for $N K X 2.5$ in atrial, ventricular, and conotruncal septation, AV valve formation, and maintenance of AV conduction.

\section{Methods}

Informed consent was obtained from all participants in accordance with the Medical University of South Carolina institutional review board for Human Research. Subjects were evaluated by history, review of medical records, physical examination, 12-lead electrocardiogram (ECG), and 2-dimensional transthoracic echocardiography with colorflow Doppler interrogation using standard views.
Transesophageal echocardiography, cardiac catheterization, electrophysiology study, and/or cardiac surgery had been performed in some individuals. Clinical studies were performed without knowledge of genotype.

\section{Patient groups}

Rationale for patient groups. Schott et al. (19) identified 19 individuals in 4 kindreds with $N K X 2.5$ mutations. Genotype-positive individuals had first-, second-, or third-degree AV block, and 84\% had ASD. Thus, some individuals without a congenital heart defect developed AV conduction block. We hypothesized that NKX2.5 mutation may cause these idiopathic AV blockages. In addition, some individuals within the 4 kindreds (that had not been genotyped) had a history of tetralogy of Fallot and ventricular septal defect. Based on these observations, we hypothesized that other forms of congenital heart disease, including tetralogy of Fallot, may result from $N K X 2.5$ mutation. To further understand the role of $N K X 2.5$ in human cardiac morphogenesis, we studied 3 proband groups.

Group 1: Cardiovascular malformation and first-degree $A V$ block. Five individuals who underwent preoperative evaluation of congenital cardiovascular malformation were identified to have first-degree AV block. Family history assessment demonstrated autosomal dominant transmission of congenital heart disease and/or AV conduction disturbance in 4 kindreds (Figure 1).
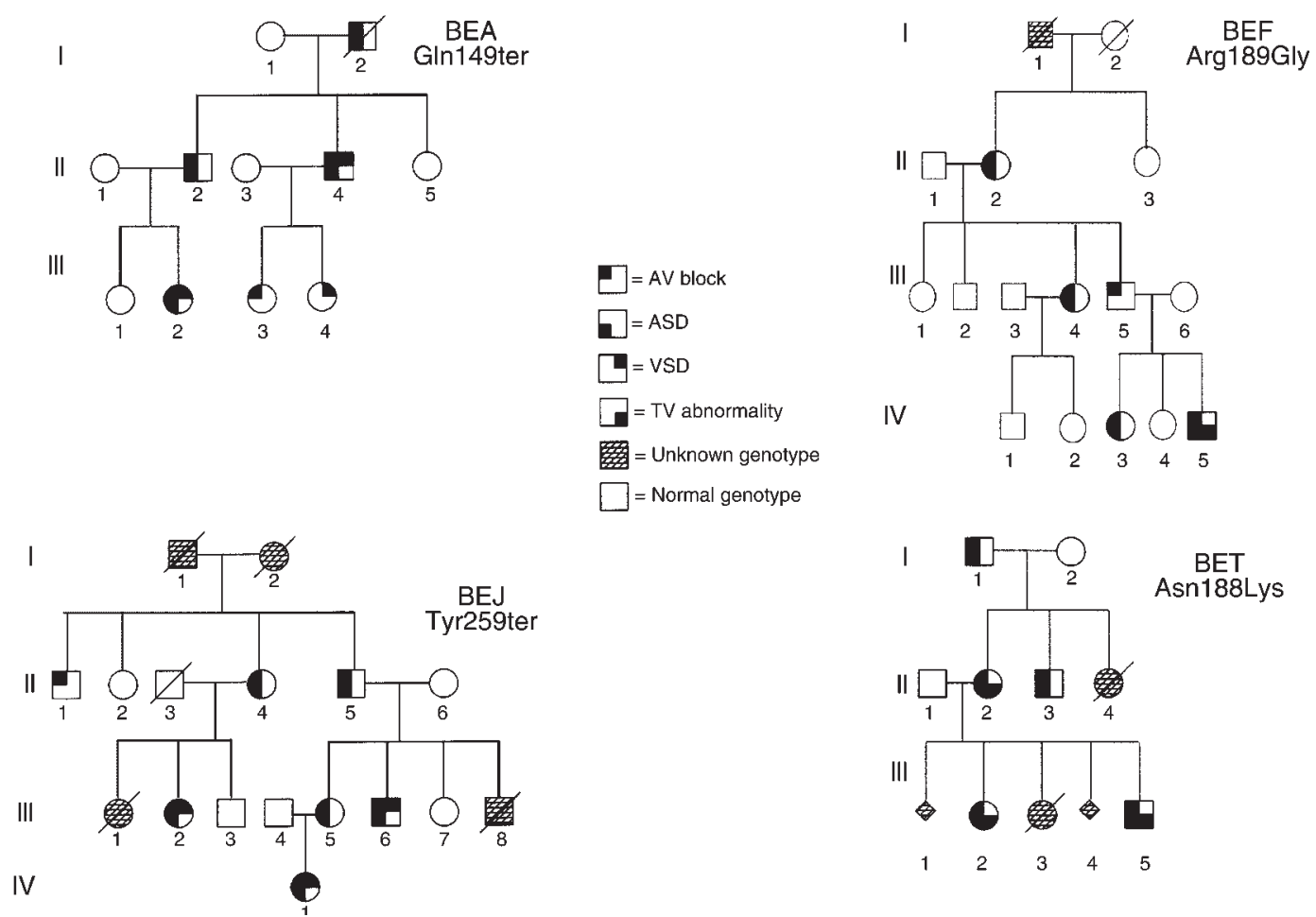

Figure 1

Pedigree of familial congenital heart disease. The mutation found in each family is shown under the pedigree identification. Men are denoted by squares, women by circles. Closed symbols denote individuals with a NKX2.5 mutation. To illustrate variation in phenotype, the symbol is divided into quadrants indicating the presence of AV block, ASD, VSD, or tricuspid valve (TV) abnormality (including Ebstein's anomaly). Open symbols denote normal genotype. Hatched symbols denote individuals with unknown genotype status. 
Table 2

Genotype/Phenotype summary

\begin{tabular}{|c|c|c|c|c|c|c|c|c|c|c|}
\hline Identification & Mutation & Site & Confirm & $\begin{array}{l}\text { Genotype } \\
\text { positive }\end{array}$ & $\begin{array}{c}\text { AV } \\
\text { block }\end{array}$ & ASD & $\begin{array}{l}\text { VSD cono- } \\
\text { ventricular }\end{array}$ & $\begin{array}{c}\text { VSD } \\
\text { muscular }\end{array}$ & $\begin{array}{l}\text { TV } \\
\text { abn }\end{array}$ & Other \\
\hline \multicolumn{11}{|l|}{ Group 1} \\
\hline BEA & C554T, Gln149ter & Homeodomain & ASO & 6 & 5 & 4 & $1^{\mathrm{A}}$ & $2^{\mathrm{E}}$ & 0 & \\
\hline BEF & C674G, Arg189Gly & Homeodomain & Abolish Msp I site & 5 & 5 & $4^{\mathrm{D}}$ & 0 & 0 & 1 & 3- LV $\downarrow$ \\
\hline BEJ & C886A, Tyr259ter & $3^{\prime}$-coding region & Abolish Mae III site & 7 & 7 & 6 & $2^{B, C}$ & 1 & 0 & \\
\hline BEP & A681G, Tyr191Cys & Homeodomain & ASO & 1 & 1 & 1 & 1 & 0 & 0 & \\
\hline BET & C673A, Asn188Lys & Homeodomain & Abolish Msp I site & 5 & 5 & 5 & 0 & 0 & $3^{F}$ & $1-L V \downarrow$ \\
\hline \multicolumn{11}{|l|}{ Group 2} \\
\hline CHB 3-11 & Int 1DSG+1T & Splice site & Create Hph I site & 1 & 1 & 0 & 0 & 0 & 0 & \\
\hline \multicolumn{11}{|l|}{ Group 3} \\
\hline TOF 7 & C182T, Arg25Cys & $5^{\prime}$-coding region & Create Bsg I site & 1 & 0 & 0 & $1^{\mathrm{A}}$ & 0 & 0 & \\
\hline Subtotal & & & & 26 & 24 & 20 & 5 & 3 & 4 & 4 \\
\hline \multicolumn{11}{|l|}{ Reference 19} \\
\hline MXP, MBF & C642T, Thr178Met & Homeodomain & Create Nla III site & 12 & 12 & 11 & 0 & 0 & 0 & \\
\hline MBT & C618T, Gln170ter & Homeodomain & Create $\mathrm{Bfa}$ I site & 4 & 4 & 2 & 0 & 0 & 0 & 1- LV $\uparrow$ \\
\hline MBX & C701T, Gln198ter & $3^{\prime}$-coding region & ASO & 3 & 3 & 3 & 0 & 0 & 0 & \\
\hline Subtotal & & & & 19 & 19 & 16 & 0 & 0 & 0 & 1 \\
\hline
\end{tabular}

ASD, secundum atrial septal defect; ASO, allele-specific oligonucleotide hybridization; AV, atrioventricular; LV $\downarrow$, reduced left ventricular function; LV $\uparrow$, left ventricular hypertrophy; TOF, tetralogy of Fallot; TV, abn tricuspid valve abnormality; VSD, ventricular septal defect. A, adenine; C, cytosine; G, guanine; T, thymine; Arg, arginine; Cys, cysteine; Gln, glutamine; Gly, glycine; Lys, lysine; Met, methionine; Thr, threonine. ${ }^{A}$ VSD associated with tetralogy of Fallot; ${ }^{B}$ VSD

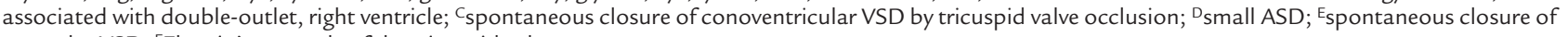
muscular VSD; FEbstein's anomaly of the tricuspid valve.

Group 2: Idiopathic second- or third-degree AV block. We identified 10 probands who had been treated with a pacemaker for idiopathic second- or third-degree AV block. None had a previous history of any other cardiac surgery or other evidence of heart disease. Electrophysiologic study was performed in 3 probands. None of the mothers of the 10 probands had autoantibodies to SSA/Ro or SSB/La ribonucleoprotein (20). However, a history of heart disease under the age of 40 years in at least 1 other family member was identified in 6 cases and included AV conduction disturbance, atrial fibrillation, or sudden death.

Group 3: Tetralogy of Fallot without del22q11. Twenty probands with tetralogy of Fallot were identified from the St. Louis Children's Hospital cytogenetic laboratory database. These cases were selected from 159 cases evaluated from August 1993 to June 1995 (21). A chromosome 22q11 deletion (del22q11) was identified in $14 \%$ who underwent fluorescent in situ hybridization (FISH) testing with the N25 cosmid probe. To exclude del22q11 as a cause of tetralogy of Fallot, a group of 20 patients with tetralogy of Fallot and a negative result for del22q11 were selected at random for evaluation of a NKX2.5 mutation. Anatomic diagnosis was verified from echocardiographic records, cardiac catheterization studies, and findings at cardiac surgery. All had undergone definitive surgery for tetralogy of Fallot before the age of 3 years. In 3 cases, there was a family history of other congenital heart disease. DNA from other family members was not available for evaluation, as the DNA samples of the 20 tetralogy of Fallot patients were received anonymously.

\section{Genetic studies}

Cytogenetic studies. FISH testing had been performed on 20 patients with tetralogy of Fallot using the N25 cosmid probe (D22S75) for the deletion in 22q11.2 and the control ( $\mathrm{pH} 17$ ) probe (D22S39), which tags the 22q13.3 band (Oncor Inc., Gaithersburg, Maryland, USA). Scoring and validation with these probes was described previously (21). Standard cytogenetic analysis was performed after ethidium bromide exposure of cultured skin fibroblasts or blood lymphocytes stimulated by either phytohemagglutinin or pokeweed mitogen.

Molecular genetic studies. The gene encoding the homeobox transcription factor, $N K X 2.5$, contains 2 exons that encode a 324-amino acid protein $(7,22)$. Primers were designed to amplify both exons and the intervening intron in a single PCR (Table 1) using genomic DNA isolated from peripheral lymphocytes or cultured fibroblasts using Puregene reagents (Gentra Systems Inc., Minneapolis, Minnesota, USA). In brief, 100 ng of genomic DNA was amplified in a $25-\mu \mathrm{L}$ volume containing $40 \mathrm{ng}$ of each oligonucleotide primer (1F and 4R); $200 \mu \mathrm{mol} / \mathrm{L}$ each of deoxyadenosine triphosphate, deoxycytidine triphosphate, deoxyguanosine triphosphate, and deoxythymidine triphosphate; $5 \mu \mathrm{L}$ of Q solution (QIAGEN Inc., Valencia, California, USA) and Taq polymerase. The samples were denatured for 2 minutes at $95^{\circ} \mathrm{C}$, and then processed through 35 cycles including denaturation at $94^{\circ} \mathrm{C}$ for $10 \mathrm{~seconds}$, primer annealing at $65^{\circ} \mathrm{C}$ for $30 \mathrm{sec}-$ onds, and primer extension at $68^{\circ} \mathrm{C}$ for 2 minutes. The amplified products were electrophoresed on $1 \%$ agarose gels. Gel-isolated PCR products were used in sequencing 

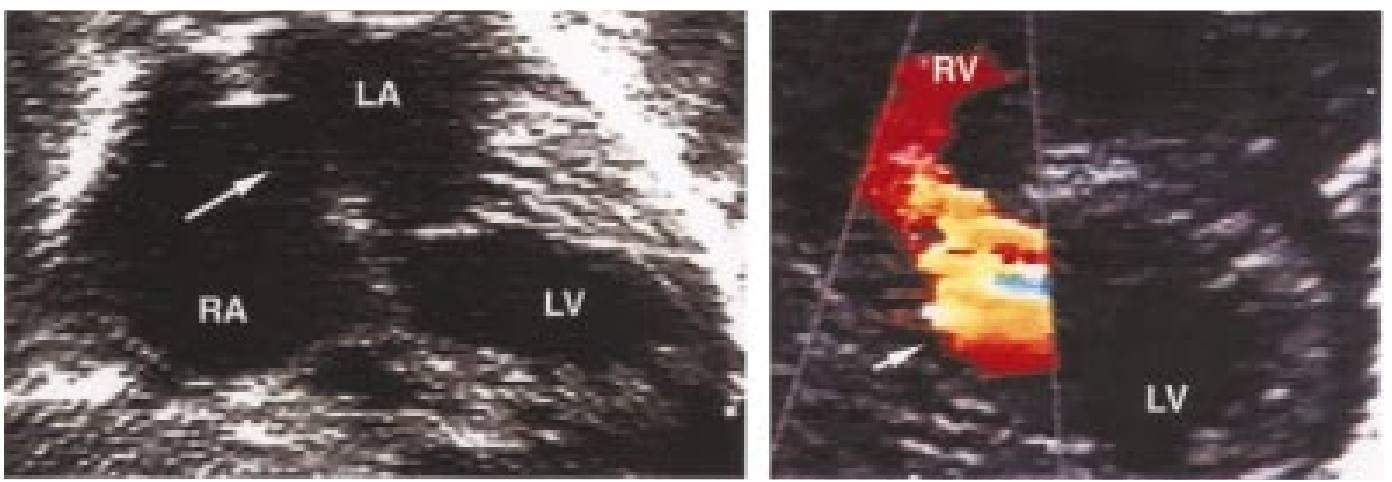

\section{Figure 2}

Echocardiogram/Doppler showing secundum ASD and posterior muscular VSD (BEA III-2). Subcostal view shows large secundum ASD (left). The parasternal short axis view shows posterior muscular VSD with left ventricle to right ventricle blood flow on color Doppler interrogation (right). The VSD spontaneously closed during the first 6 months of life; the ASD was surgically closed at 10 months of age. LA, left atrium; LV, left ventricle; RA, right atrium; RV, right ventricle.

reactions performed in the presence of fluorescencelabeled dideoxynucleotides and additional primer designed to sequence the coding region (Table 1). Products were fractionated on an ABI377 automated DNA sequencer and analyzed using Auto Assembler (PerkinElmer Biosystems, Foster City, California, USA). and SEQMAN (DNASTAR Inc., Madison, Wisconsin, USA).

\section{Results}

Mutation analysis. As summarized in Table 2, 7 NKX2.5 mutations were identified in 7 probands. Numbering for all the mutations starts at the adenine nucleotide (A) in the ATG initiation codon (8). Five mutations changed a restriction enzyme site allowing independent confirmation; 2 mutations were confirmed by allelespecific oligonucleotide hybridization (23). One missense mutation is predicted to result in codon change in the $5^{\prime}$-coding region (Arg25Cys), and 3 missense mutations are predicted to result in codon changes in the homeodomain (Asn188Lys, Arg189Gly, and Tyr191Cys). Two single nucleotide changes are predicted to produce a termination codon in the homeodomain (Gln149ter) or in the $3^{\prime}$-coding region (Tyr259ter). One mutation changed the first nucleotide of the intron (the splice-donor site) (Int 1DSG+1T).

These NKX2.5 sequence variants were considered mutations based on their cosegregation with cardiovascular disease, the significant change each was predicted to cause in the protein structure of NKX2.5, and their absence in more than 100 chromosomes derived from unrelated normal subjects.

In addition to the 7 mutations, an $\mathrm{A} 172 \mathrm{G}$ polymorphism or silent mutation (Glu21Glu) was observed in 52 control samples such that $33 \%$ were AA, $36 \%$ were AG, and $31 \%$ were GG. The polymorphism creates a BpmI restriction enzyme site allowing independent confirmation.

\section{Clinical features of genotype-positive individuals}

Group 1: Cardiovascular malformations and first-degree $A V$ block. Six members from 3 generations of Family BEA had a Gln149ter mutation (Figure 1). Three individuals had an ASD (Table 2). I-2 was diagnosed with ASD and advanced second-degree AV block in adulthood and underwent surgical closure and pacemaker implantation at age 25 years. II-2 underwent ASD closure at 6 years of age, and at age 30 years II-2 has marked first degree AV block. III-2 underwent ASD closure at age 10 months for failure to thrive, a muscular VSD that spontaneously closed had been noted earlier in life (Figure 2). II-4 was diagnosed with tetralogy of Fallot and ASD in infancy. II-4 was treated with a Blalock-Taussig shunt at 18 months and definitive surgery at age 6 years. A pacemaker was implanted at age 8 years. III-3 had ECG evidence of first-degree AV block at age 5 years. Her sister, III-4, had echocardiographic documentation of a midmuscular VSD that spontaneously closed in infancy and a normal ECG at age 3 years.

Five members from 3 generations of Family BEF had an Arg189Gly mutation (Figure 1). Based on echocardiographic evidence of reduced ventricular function and rhythm disturbances (including complex ventricular ectopy, atrial flutter, atrial fibrillation, and/or advanced second-degree AV block), II-2, III-4, and III-5 were considered to have a familial cardiomyopathy; all were treated with a pacemaker. Transesophageal echocardiography revealed a small ASD in II-2 and III4. IV-5 underwent surgical closure of ASD in 1998 at age 7 years. Her brother, IV-3, had echocardiographic evidence of a large ASD with a little left to right shunt. Findings at cardiac catheterization and surgery demonstrated a small tricuspid valve. Both IV-3 and IV-5 have prolonged PR interval. I-1 was not genotyped, but he had a history of recurrent syncope and died suddenly in 1947 at age 52 years.

Seven members of Family BEJ had a Tyr259ter mutation (Figure 1). II-4 and II-5 had undergone ASD closure and pacemaker implantation at age 27 and 19 years, respectively. II- 1 had advanced second-degree AV block and ventricular dysrhythmias. III-2 underwent ASD closure at age 6 years; a perimembranous VSD was spontaneously occluded by tricuspid valve tissue. III-5 underwent ASD closure at age 13 years. At 15 months 
of age, III-6 underwent closure of an ASD and closure of a VSD associated with double-outlet right ventricle by baffling the left ventricular outflow through the VSD into the aorta. IV-1 was diagnosed in infancy with ASD and "Swiss cheese" VSD. Clinical information was available on 2 individuals who were not genotyped. III1 died of pneumonia complicated by severe heart failure resulting from large ASD and VSD in 1967, at 22 months of age. III-8 died in 1979 at 17 days of age after surgery for coarctation of the aorta; he also had ASD and VSD. All affected family members had ECG evidence of prolonged AV conduction.

BEP II-3 had a Tyr191Cys mutation. The father, mother, and siblings did not carry this mutation, indicating that it is de novo (pedigree not shown). First-degree AV block, ASD, and VSD were diagnosed in infancy. Surgical closure of ASD and VSD was performed at 6 months of age because of medically refractory heart failure.

Five members of family BET had an Asn188Lys mutation (Figure 1). I-1 was diagnosed with ASD at age 37 years, and he underwent ASD closure and pacemaker implantation at the age of 53 years. Medically refractory heart failure led to heart transplantation at age 60 years. II- 2 was followed for many years with a diagnosis of Ebstein's anomaly of the tricuspid valve; she underwent ASD closure at age 37 years. II- 3 underwent ASD closure at age 8 years. III-2 has Ebstein's anomaly and underwent ASD closure at age 2 years (Figure 3). III-5 underwent ASD closure at age 7 years and was noted to have a tethered tricuspid valve. All affected family members have evidence of abnormal
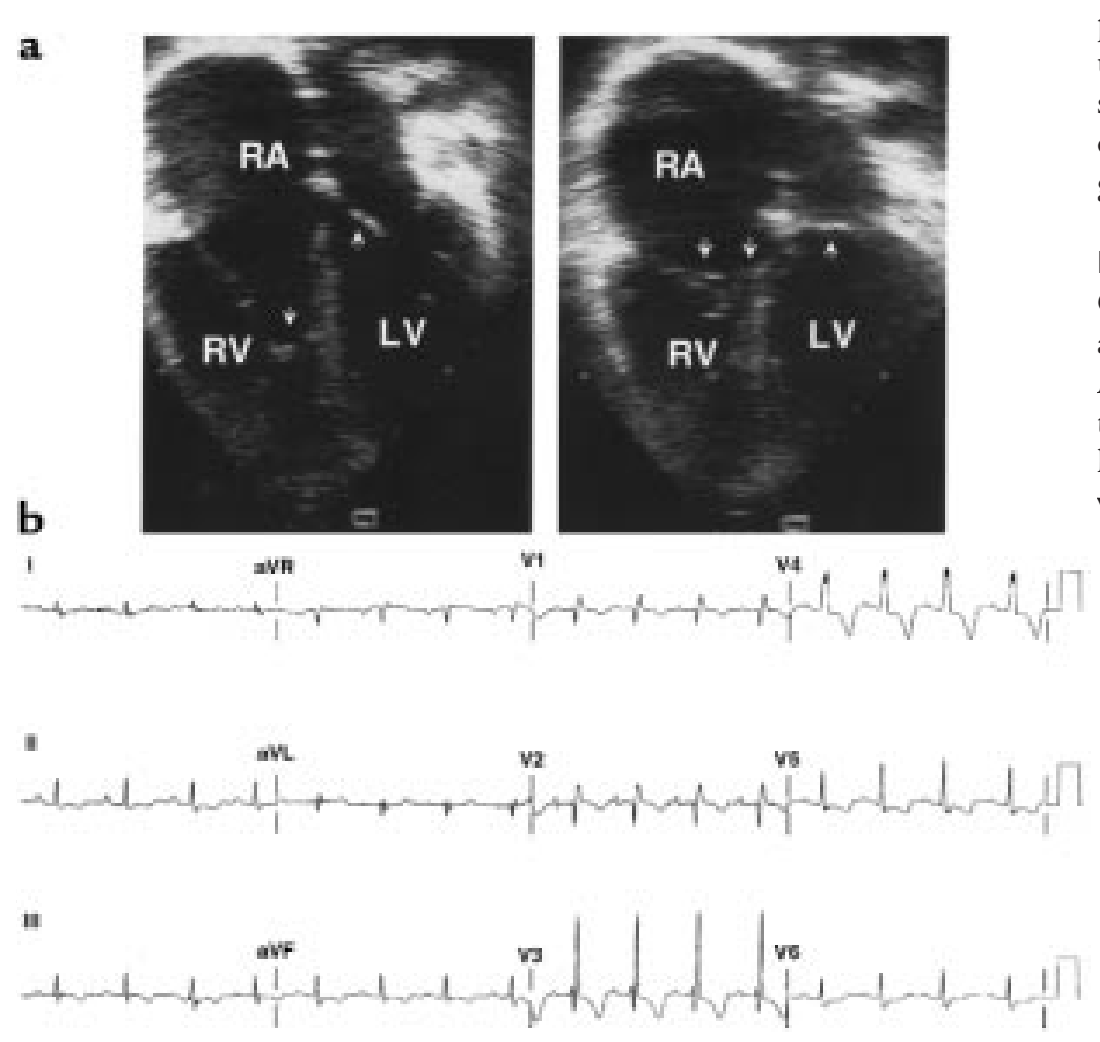

AV conduction. In addition, III-2 and III-5 have right bundle branch block, commonly seen in Ebstein's anomaly. Clinical data are available for several individuals who could not be genotyped. II-5 was a "blue baby" who died in 1956 at 13 months of age. III- 1 and III-4 were spontaneous first trimester abortions. III-3 was delivered prematurely after a 36-week gestation by emergency Cesarean section for placenta abruption; death occurred shortly after birth.

Group 2: Idiopathic second-or third-degree AV block. CHB3 has a splice site mutation in exon 1 (Int 1DSG+1T, Figure 4); his was the only NKX2.5 mutation among the 10 probands tested. No phenotypic feature of this individual distinguished him from others in this group. He presented at 12 years of age with a 1-year history of recurrent syncope. Advanced second-degree AV block was identified on ECG (not shown); no other cardiac abnormalities were noted. Pacemaker implantation was performed. Evaluation of his mother and 2 younger brothers was normal, and none carried the mutation (pedigree not shown). His father (not genotyped) had died suddenly - presumably because of arrhythmias in 1984 at 29 years of age. His heart weight was $300 \mathrm{~g}$, and no major abnormalities were noted at autopsy.

Group 3: Tetralogy of Fallot negative for del22q11. TOF7 has an Arg25Cys mutation. Hers was the only NKX2.5 mutation of 20 probands tested. There was no phenotypic feature of this individual that distinguished her from other group members, but unlike BEA II-4, who also had tetralogy of Fallot, she did not have AV block or an ASD. She underwent surgery at 12 months of age for typical tetralogy of Fallot and 2 small muscular VSDs. She had small pulmonary arteries with areas of peripheral narrowing, and she had undergone balloon dilation on 2 occasions. There was no family history of other heart disease; we were not able to genotype other family members.

\section{Discussion}

Observations in this study provide additional evidence for a role of NKX2.5 beyond early cardiac progenitor commitment; the coinheritance of heterozygous NKX2.5 mutations with various congenital heart defects sug-

\footnotetext{
Figure 3

Echocardiogram and ECG characteristics of Ebstein's anomaly (BET III-2). (a) Four chamber echocardiogram during diastole (left) and systole (right); arrows indicate location of tricuspid and mitral valve (left and right). A saillike anterior tricuspid valve leaflet is evident in diastole; inferior displacement of the septal leaflet is evident in systole. RA, right atrium; $\mathrm{RV}$, right ventricle; LV, left ventricle. (b) ECG shows first-degree AV block and right bundle branch block (bottom).
} 


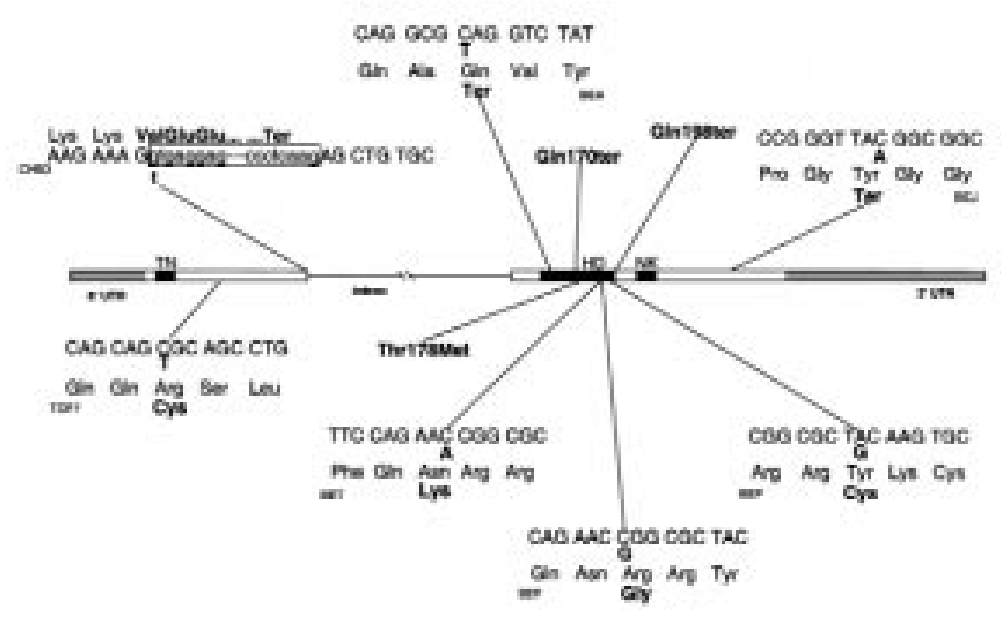

Figure 4
Diagram of the NKX2.5 gene showing 10 heterozy-
gote mutations. Three mutations were reported pre-
viously (Gln170ter, Thr $178 \mathrm{Met}$, Gln198ter) (19).
The region enclosed by boxes indicates exon 1 (left)
and exon 2 (right); the horizontal line indicates the
intron. Shaded boxes indicate the 5' and 3' untrans-
lated regions (UTR), respectively. The white box
denotes the coding region, and the black boxes indi-
cate the conserved TN domain, homeodomain
(HD), and NK domain, respectively, within the cod-
ing region. Nonsense mutations are indicated above
and missense mutations below the gene diagram.

gests that this transcription factor contributes to diverse cardiac developmental pathways including atrial, ventricular, and conotruncal septation, AV conduction, and $A V$ valve formation. As described previously, AV block and ASD are common in kindreds in whom congenital heart disease is due to NKX2.5 mutation (19). Additionally, NKX2.5 mutation causes AV block without associated congenital heart defects, and, as demonstrated in the patients we studied, AV block was the principal clinical finding in $23 \%$ of genotype-positive individuals. Further, based on results of the present study, NKX2.5 mutation may account for a clinically significant portion of idiopathic AV block.

However, cardiovascular defects resulting from NKX2.5 mutations extend beyond AV block and ASD. For example, VSD was present in $31 \%$ of genotype-positive individuals, including conoventricular VSD associated with tetralogy of Fallot and double-outlet, right ventricle and muscular VSD that closed spontaneously in infancy (24). Results of this study suggest that in individuals without a del22q11, NKX2.5 mutations may account for a significant portion of conotruncal defects (25). Abnormalities of the tricuspid valve were also present (15\%), and for the first time, we have identified a genetic cause of Ebstein's anomaly. Based on the diversity of cardiac phenotypes, we hypothesize that many additional forms of congenital heart disease may result from NKX2.5 mutations. The small sample size we studied does not allow estimation of a portion of cardiac anomalies resulting from $N K X 2.5$; our study is further limited by concentration on mutations in coding sequences only.

The 7 NKX2.5 mutations described in this study, and the 3 reported previously, are most often located in the region encoding the homeodomain and less often in the $5^{\prime}$ and $3^{\prime}$ ends of the gene (Figure 4). Of the 10 total mutations, 5 are predicted to result in truncated proteins that have the potential for dominant-negative or deleterious gain of function effects. The other 5 missense mutations are predicted to alter single, highly conserved amino acids in the homeodomain. The mechanism by which these mutations produce diverse cardiac malformations remains to be determined. Pauli et al. (26) reported a patient with haploinsufficiency of
NKX2.5 (CSX) resulting from a distal chromosome $5 \mathrm{q}$ deletion that manifested ASD, AV block, and ventricular noncompaction. Because of the large number of our patients who had ASD and AV block, many of the mutations we have identified may function as null alleles. However, some specific genotype/phenotype correlation can be speculated. For example, the 4 individuals with tricuspid valve abnormalities, including Ebstein's anomaly, come from 2 families where missense mutations in adjacent codons (Asn188Lys and Arg189Gly) were identified.

Schott et al. (19) described 2 families with an identical NKX2.5 mutation and an identical haplotype indicating common ancestry. In subjects we evaluated, each proband had a novel mutation. Out of the 7 mutations, 3 occurred in CPG islands, which have an elevated mutation rate compared with other dinucleotides (27). The mutation in BEP II- 11 is a sporadic occurrence as neither parent carries the Tyr191Cys mutation. TOF7 and CHB3 may be examples of sporadic occurrence, but we could not confirm this possibility because both parents could not be genotyped.

Previous studies have identified $N k \times 2.5$ as an upstream regulator and/or transcriptional activator of other genes expressed during cardiac development including Hand1 (eHand) $(9,11)$, myocyte enhancer factor-2 (MEF2) $(9,12)$, myosin light chain 2V (MLC2V) (9), atrial natriuretic factor gene $(9,13)$, brain natriuretic peptide (BNP) (9), $\alpha$-cardiac actin gene (14), cardiac ankyrin repeat protein gene (15), N-myc (9), and MSX2 (9). Nkx2.5 binding to target DNA may occur in conjunction with other factors including GATA-4 $(16,18)$ and serum response factor (14). The demonstration of synergistic transcriptional activation mediated by Nkx2.5 and GATA-4 (28), and the elucidation of the HoxB1-Pbx1 and Ultrabithorax-Extradenticle structure of heteromeric complexes $(29,30)$, suggests ways in which mutations may alter the specificity and affinity of DNA binding, while also disrupting the tightly regulated spatial and temporal gene expression that is essential during cardiac development.

The severe phenotypes associated with heterozygous NKX2.5 mutations in humans are surprising, given the 
phenotypes of heterozygous mutations modeled in other organisms. For example, ablation of $N k \times 2.5$ in mice was embryonic lethal, but heart defects were not observed in heterozygous mutant mice $(8,9)$. The differences between mice and humans may reflect a bias resulting from observing only severely affected humans. Alternatively, these differences may reflect different cardiac development in mice and humans, genetic redundancy in humans, and/or unrecognized phenotypes in heterozygous mutant mice.

Diverse cardiac malformations are recognized in other monogenic human disorders, e.g., heterozygous TBX5 mutations in Holt-Oram syndrome (31-33), as well as gene-targeted mice, e.g., heterozygous mutations in retinoic $X$ receptor-deficient mice (34). Although variable expressivity is not a feature predicted by classic embryological models of cardiac development, heritable monogenic mutations can clearly cause pleiotropic cardiovascular defects. Based on results of the present studies, $N K X 2.5$ appears to be a likely candidate gene for a number of forms of cardiovascular disease in the young.

\section{Acknowledgments}

We are indebted to the members of these families for their participation. These studies would not have been possible without the skillful assistance of Linda Johnson, Barbara Roberts, Marlene Brabham, Ping Lu, and Kris Houston. This work was supported in part by National Institutes of Health grant 1 P50 HL61006-01 (to D.W. Benson) and Howard Hughes Medical Institute (to J.G. Seidman and C.E. Seidman).

1. Olson, E.N., and Srivastava, D. 1996. Molecular pathways controlling heart development. Science. 272:671-676.

2. Harvey, R.D. 1996. Nk-2 homeobox genes and heart development. Dev. Biol. 178:203-216.

3. Fishman, M.C., and Olson, E.N. 1997. Parsing the heart: genetic modules for organ assembly. Cell. 91:153-156.

4. Bodmer, R. 1993. The gene, tinman, is required for specification of the heart and visceral muscles in Drosophilia. Development. 118:719-729.

5. Lints, T.J., Parsons, L.M., Hartley, L., Lyons, I., and Harvey, R.P. 1993. Nkx 2-5: a novel murine homeobox gene expressed in early heart progenitor cells and their myogenic descendents. Development. 119:419-431.

6. Komuro, I., and Izumo, S. 1993. Csx: a murine homeobox-containing gene specifically expressed in the developing heart. Proc. Natl. Acad. Sci. USA. 90:8145-8149.

7. Shiojima, I., et al. 1996. Molecular cloning and characterization of human cardiac homeobox gene CSX1. Circ. Res. 79:920-929.

8. Lyons, I., et al. 1995. Myogenic and morphogenetic defects in the heart tubes of murine embryos lacking the homeo box gene Nkx2-5. Genes Dev. 9:1654-1666.

9. Tanaka, M., Chen, Z., Bartunkova, S., Yamasaki, N., and Izumo, S. 1999. The cardiac homeobox gene Csx/Nkx2.5 lies genetically upstream of multiple genes essential for heart development. Development. 126:1269-1280

10. Chen, C.Y., and Schwartz, R.J. 1995. Identification of novel DNA binding targets and regulatory domains of a murine Tinman homeodomain factor, nkx2-5. J. Biol. Chem. 270:15628-15633.

11. Biben, C., and Harvey, R.P. 1997. Homeodomain factor Nkx2-5 controls left/right asymmetric expression of bHLH gene eHand during murine heart development. Genes Dev. 11:1357-1369.

12. Gajewski, K., Kim, Y., Lee, Y.M., Olson, E.N., and Schulz, R.A. 1997. D-mef2 is a target for Tinman activation during Drosophilia heart development. EMBO J. 16:515-522.

13. Durocher, D., Chen, C.-Y., Ardati, A., Schwartz, R.J., and Nemer, M. 1996. The atrial natriuretic factor promoter is a downstream target for Nkx2-5 in the myocardium. Mol. Cell. Biol. 16:4648-4655.

14. Chen, C.Y., et al. 1996. Activation of the cardiac alpha-actin promoter depends upon serum response factor, Tinman homologue, $N k \times 2-5$, and intact serum response elements. Dev. Genet. 19:119-130.

15. Zou, Y., et al. 1997. CARP, a cardiac ankyrin repeat protein is downstream in the Nkx2-5 homeobox gene pathway. Development. 124:793-804.

16. Durocher, D., Charron, F., Warren, R., Schwartz, R.J., and Nemer, M. 1997. The cardiac transcription factors $N k \times 2-5$ and GATA-4 are mutual cofactors. EMBO J. 16:5687-5696.

17. Kasahara, H., and Izumo, S. 1999. Identification of the in vivo casein kinase II phosphorylation site within the homeodomain of the cardiac tissue-specifying homeobox gene product Csx/Nkx2-5. Mol. Cell. Biol. 19:526-536.

18. Lee, Y., et al. 1998. The cardiac tissue-restricted homeobox protein Csx/ Nkx2-5 physically associates with the zinc finger protein GATA4 and cooperatively activates atrial natriuretic factor gene expression. Mol. Cell. Biol. 18:3120-3129.

19. Schott, J.-J., et al. 1998. Congenital heart disease caused by mutations in the transcription factor Nkx2-5. Science. 281:108-111.

20. Buyon, J.P., et al. 1998. Autoimmune-associated congenital heart block: demographics, mortality, morbidity and recurrence rates obtained from a national neonatal lupus registry. J. Am. Coll. Cardiol. 31:1658-1666.

21. Johnson, M.C., Hing, A., Wood, M.K., and Watson, M.S. 1997. Chromosome abnormalities in congenital heart disease. Am. J. Med. Genet. 70:292-298.

22. Turbay, D., Wechsler, S.B., Blanchard, K.M., and Izumo, S. 1996. Molecular cloning, chromosomal mapping, and characterization of the human cardiac-specific homeobox gene $h C s x$. Mol. Med. 2:86-96.

23. Benson, D.W., et al. 1996. A missense mutation in the pore region of HERG causes familial long QT syndrome. Circulation. 93:1791-1795.

24. Van Praagh, R., Geva, T., and Kreutzer, J. 1989. Ventricular septal defects: how shall we describe, name and classify them? J. Am. Coll. Cardiol. 14:1298-1299.

25. Goldmuntz, E., et al. 1998. Frequency of 22q11 deletions in patients with conotruncal defects. J. Am. Coll. Cardiol. 32:492-498.

26. Pauli, R.M., Scheib-Wixted, S., Cripe, L., Izumo, S., and Sekhon, G.S. 1999. Ventricular noncompaction and distal chromosome $5 \mathrm{q}$ deletion. Am.J. Med. Genet. 85:419-423.

27. Ketterling, R.P., Vielhaber, E., and Sommer, S. 1994. The rates of G:C-T:A and G:C-C:G transversions at CPG dinucleotides in the human Factor IX gene. Am. J. Hum. Genet. 54:831-835.

28. Shiojima, I, et al. 1999. Context-dependent transcriptional cooperation mediated by cardiac transcription factors $C s x / N k x 2.5$ and GATA-4. J. Biol. Chem. 274:8231-8239.

29. Piper, D.E., Batchelor, A.H., Chang, C.-P., Cleary, M.L., and Wolberger, C. 1999. Structure of a HoxB1-Pbx1 heterodimer bound to DNA: role of the hexapeptide and a fourth homeodomain helix in complex formation. Cell. 96:587-597.

30. Passner, J.M., Ryoo, H.D., Shen, L., Mann, R.S., and Aggarwal, A.K. 1999. Structure of a DNA-bound Ultrabithorax-Extradenticle homeodomain complex. Nature. 397:714-719.

31. Li, Q.Y., et al. 1997. Holt-Oram syndrome is caused by mutations in TBX5 a member of the Brachyury (T) gene family. Nat. Genet. 15:21-29.

32. Basson, C.T., et al. 1997. Mutations in human TBX5 cause limb and cardiac malformation in Holt-Oram syndrome. Nat. Genet. 15:30-35.

33. Basson, C.T., et al. 1999. Different TBX5 interactions in heart and limb defined by Holt-Oram syndrome mutations. Proc. Natl. Acad. Sci. USA. 96:2919-2924.

34. Gruber, P.J., et al. 1996. RXR $\alpha$ deficiency confers genetic susceptibility for aortic sac, conotruncal, atrioventricular cushion, and ventricular muscle defects in mice. J. Clin. Invest. 98:1332-1343. 